\title{
On the Zeros of Plane Partition Polynomials
}

\author{
Robert P. Boyer and Daniel T. Parry \\ Department of Mathematics \\ Drexel University \\ Philadelphia, PA 19104 \\ rboyer@drexel.edu, dtp29@drexel.edu
}

Submitted: Apr 29, 2011; Accepted: Dec 17, 2011; Published: Jan 2, 2012

Mathematics Subject Classification: Primary: 11C08 Secondary: 11M35, 30C55, 30E15

\section{Dedicated to Doron ZeIlberger on the OCCASION OF HIS SIXTIETH BIRThDAY}

\begin{abstract}
Let $P L(n)$ be the number of all plane partitions of $n$ while $p p_{k}(n)$ be the number of plane partitions of $n$ whose trace is exactly $k$. We study the zeros of polynomial versions $Q_{n}(x)$ of plane partitions where $Q_{n}(x)=\sum p p_{k}(n) x^{k}$. Based on the asymptotics we have developed for $Q_{n}(x)$ and computational evidence, we determine the limiting behavior of the zeros of $Q_{n}(x)$ as $n \rightarrow \infty$. The distribution of the zeros has a two-scale behavior which has order $n^{2 / 3}$ inside the unit disk while has order $n$ on the unit circle.
\end{abstract}

Keywords: asymptotic, phase, plane partition, polylogarithm

\section{Introduction}

Over the past ten years, many examples of natural polynomial families from combinatorics and number theory have emerged whose zeros for high degrees appear to converge to intriguing curves in the complex plane. One interesting collection of examples appears on the website [16] of Richard Stanley which includes chromatic polynomials of complete partite graphs, $q$-analogue of Catalan numbers, Bernoulli polynomials, and others.

Previous emphasis has been on polynomials all of whose zeros are real as well as on polynomials whose coefficients are unimodal or log-concave. The connection between the zeros and coefficients, of course, comes from the fact that if all the zeros are real and negative, then the coefficients are log-concave.

In [4], Boyer and Goh investigated the limiting behavior of zeros of the "partition polynomials" $F_{n}(x)$ where

$$
F_{n}(x)=\sum_{k=1}^{n} p_{k}(n) x^{k}
$$


where $p_{k}(n)$ is the number of integer partitions of $n$ with exactly $k$ parts. This is one of the examples posted by Stanley. These polynomials were also mentioned earlier in the paper "Durfee polynomials" [8] and even earlier their asymptotics on the positive real line were studied by E.M. Wright in [18].

There is a principle from equilibrium statistical mechanics that the zeros of a sequence of polynomials accumulate along the boundaries of the regions where their sense of the asymptotics change (see [3]). For the partition polynomials, the asymptotics inside the open unit disk $\mathbb{D}$ are governed by a sequence of functions $\left(L_{k}(x)\right)$ where $L_{k}(x)=L_{1}\left(x^{k}\right) / k$ with $L_{1}(x)=\sqrt{L i_{2}(x)}$. Here $L i_{p}(x)$ is the polylogarithm function given by the series $\sum_{n=1}^{\infty} x^{n} / n^{p},|x|<1$. When the polynomials are normalized as $\ln \left[F_{n}(x)\right] / \sqrt{n}$, their normalized form has a continuous limit, say $L(x)$, in the unit disk. The behavior of the zeros in the limit is governed by the regions of analyticity of $L(x)$. We borrow a term from statistical mechanics to describe these regions. For $m \geq 1$, we say a region is a $m$-phase if it is the set of points $x$ where $\Re\left[L_{m}(x)\right]$ dominates the other terms of the sequence; that is,

$$
R(m):=\left\{x \in \mathbb{D}: \Re\left[L_{m}(x)\right]>\Re\left[L_{j}(x)\right], j \neq m\right\} .
$$

(See Definition 4 below.) For the partition polynomials there are only three distinct $m$ phases: $R(1), R(2)$, and $R(3)$. The limit function $L(x)$ restricted to these $m$-phases is analytic and the polynomial zeros accumulate on their boundaries.

In [4], Boyer and Goh asked what is the behavior of the zeros for polynomials $\left(Q_{n}(x)\right)$ associated with the plane partitions of $n$. Recall that a plane partition $\pi=\pi_{i j}$ is an array of nonnegative integers that is weakly decreasing along each row and column with total sum $n=\sum_{i, j} \pi_{i j}$. The trace of $\pi$ is simply $\sum_{i} \pi_{i i}$, the sum of its diagonal entries. Let $p p_{k}(n)$ denote the the number of plane partitions of $n$ with trace $k$. Then the polynomial $Q_{n}(x)$ is given by

$$
Q_{n}(x)=\sum_{k=1}^{n} p p_{k}(n) x^{k}
$$

which we call the plane partition polynomial. In outline form, the main structural features that appear in [4] continue to hold for plane partitions as well. Inside the unit disk $\mathbb{D}$, there is a new sequence $\left(L_{k}(x)\right)$ to describe the asymptotics where $L_{1}(x)=\sqrt[3]{2 L i_{3}(x)}$ and $L_{k}(x)=L_{1}\left(x^{k}\right) / k$. For the plane partition polynomials, there are just two $m$-phases with $m=1,2$. So $D$ is the union of $R(1)$ and $R(2)$ together with their boundaries. The zeros of $Q_{n}(x)$ accumulate along the boundaries of $R(1)$ and $R(2)$ just as for the partition polynomials but there is another family that consists of a segment along the negative real axis that arises because of a branch cut for $L_{1}(x)$. This last family of zeros exhibit a new behavior of having a nonuniform density although being distributed on a line segment.

This paper clarifies the work initiated in [4] and identifies the key structural elements needed to determine the asymptotics and the limiting behavior of the zeros. We expect that other natural polynomial families associated with partitions can be analyzed with these methods when their generating function has an infinite product form

$$
G(x, q)=\prod_{n=1}^{\infty} \frac{1}{\left(1-x q^{n}\right)^{\alpha_{n}}}, \quad \alpha_{n} \geq 0 .
$$


When $\alpha_{n}=1$ for all $n$, we recover the partition polynomials while $\alpha_{n}=n$, we get the plane partition polynomials $Q_{n}(x)$. Perhaps the next accessible examples are the partitions whose parts lie in some fixed residue class, say $b$, modulo $m$, so $\alpha_{n}=1$ if $n \bmod m=b$ and is 0 otherwise, and case when the exponents $\alpha_{n}=n^{s}, s>1$.

We thank the referee for a careful reading of the paper and for suggesting the proof of Lemma 30.

\section{$2 \quad$ Statement of Results}

\subsection{Informal Overview}

We study two versions of the limiting behavior of the zeros of the plane partition polynomials. The first is the zero attractor $A$ that consists of all the accumulation points of the zeros of $Q_{n}(x)$. The second is the asymptotic zero distribution which is the measure, supported on $A$, given as the limit of normalized counting measures of the zeros of $Q_{n}(x)$ with normalizations $n^{\alpha}, \alpha=2 / 3$ or 1 .

The calculation of the zero attractor requires knowing the limits $\frac{1}{n^{\alpha}} \ln \left|Q_{n}(x)\right|$ as $n \rightarrow$ $\infty$ for both normalizations $\alpha=1$ and $\alpha=2 / 3$. We denote these limits by $\Phi_{\alpha}(x)$. In section 3, we find that $\Phi_{1}(x)$ equals $\ln |x|$ outside the unit disk $\mathbb{D}$ and is 0 inside by means of asymptotics built directly from the generating function for the polynomials $\prod_{n=1}^{\infty}\left(1-z q^{n}\right)^{-n}$. For $\alpha=2 / 3$, the asymptotics are more subtle that are needed to find these limits. We describe in subsection 3.2 two open connected regions $R(1)$ and $R(2)$ whose union is dense inside the unit disk. On their union $R(1) \cup R(2), Q_{n}(x)$ is asymptotic to an expression of the general form

$$
n^{-2 / 3} a(x) \exp \left(c n^{2 / 3} L_{k}(x)\right), \quad x \in R(k), k=1,2,
$$

where $c>0, L_{k}(x)$ is an analytic function on $R(k), k=1,2$, and $L_{1}(x)$ and $L_{2}(x)$ are not analytic continuations of each other. Then we find that the limit $\Phi_{2 / 3}(x)$ equals $\ln \left|L_{k}(x)\right|$, $x \in R(k)$.

We can rephrase informally the result of Sokal (Theorem 13) that the zero attractor $A$ consists of the points of non-differentiability of the limit functions $\Phi_{1}(x)$ and $\Phi_{2 / 3}(x)$. For $\alpha=1$, the set of non-differentiability of $\Phi_{1}(x)$ is the unit circle $S^{1}$ since $\Phi_{1}(x)$ is zero inside the unit disk and $\ln |x|$ outside.

For $\alpha=2 / 3$, the contributions to the zero attractor are more intricate. Points of non-differentiability of $\Phi_{2 / 3}(x)$ include the boundary points between the two regions $R(1)$ and $R(2)$ which we can show is given by $\Re\left[L_{1}(x)\right]=\Re\left[L_{2}(x)\right]$. For points in $R(1), \Phi_{2 / 3}(x)$ equals the harmonic function $\Re\left[L_{1}(x)\right]$ while for $x$ in $R(2), \Phi_{2 / 3}(x)$ becomes $\Re\left[L_{2}(x)\right]$. Hence, by the identity principle for harmonic functions, these boundary points lie in the zero attractor.

We also prove that zero attractor contains the interval $\left[x^{*}, 0\right]$ along the negative real axis that reflects the existence of a branch cut for $L_{1}(x)$. So the total contribution to $A$ is $\gamma \cup \bar{\gamma} \cup\left[x^{*}, 0\right] \cup S^{1}$ where $\gamma$ (see Figure 2) is the portion of the boundary of $R(1)$ in the upper half-plane. See Figure 3 for a full plot of $A$. 

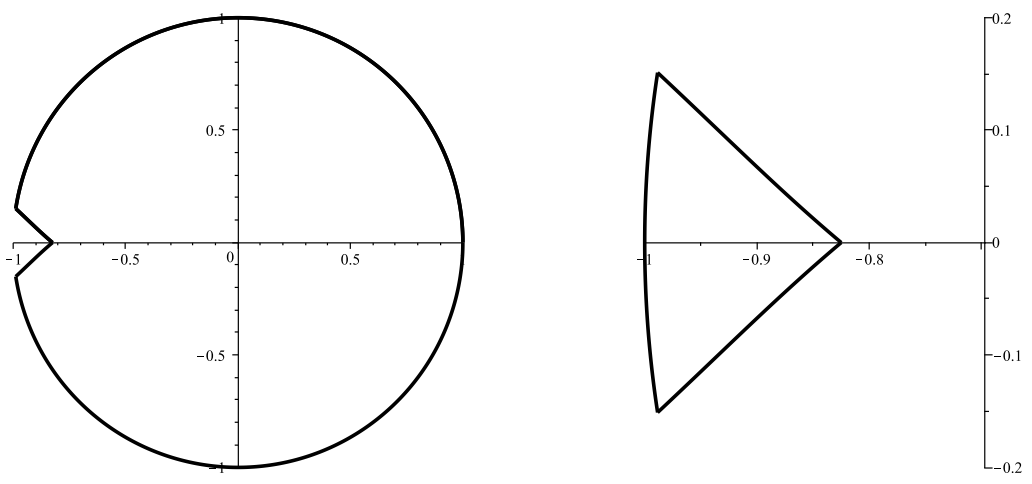

Figure 1: On the left is region $R(1)$ while on the right is the region $R(2)$. Their common boundary is $\gamma \cup \bar{\gamma}$.

The construction of the asymptotic zero distribution $\nu_{Z}$ of order $n^{2 / 3}$ needs to be carried out separately on each of the sets $\gamma, \bar{\gamma}$, and $\left[x^{*}, 0\right]$. In subsection 5.1, we focus on the contribution to $\nu_{Z}$ supported on $\gamma$. By [12] (see Theorem 27 below), if there is a neighborhood $U$ of $\gamma$ where the asymptotics for $Q_{n}(x)$ satisfy several conditions which include the existence of a conformal mapping $\psi$ between $U$ and a neighborhood of an arc of the unit circle, then $\nu_{Z}=\mu \circ \psi$ where $\mu$ is Lebesgue measure on the unit circle.

For $\gamma$, the natural choice for the mapping $\psi$ is $\exp \left(\frac{3}{2}\left(L_{2}(x)-L_{1}(x)\right)\right.$. There are several difficulties to overcome in proving that $\psi$ indeed is conformal that require techniques from univalent function theory [10].

In subsection 5.2, we found it necessary to adapt the proof of Theorem 27 in order to determine the portion of the asymptotic zero distribution $\nu_{Z}$ supported on the interval $\left[x^{*}, 0\right]$.

\subsection{Detailed Summary}

We begin with the formal definitions of the zero attractor and the asymptotic zero distribution.

Definition 1. Let $Z\left(Q_{n}(x)\right)$ denote the finite set of zeros of the polynomial $Q_{n}(x)$. Then the zero attractor $A$ of the polynomial sequence $\left(Q_{n}(x)\right)$ is the limit of $Z\left(Q_{n}(x)\right)$ in the Hausdorff metric $d$ on the non-empty compact subsets $K$ of $\mathbb{C} \cup$ " $\infty$." [5]

Definition 2. The asymptotic zero distribution $\mu$ of order $n^{\alpha}, 0<\alpha \leq 1$, for a sequence $\left(Q_{n}(x)\right)$ of polynomials is the measure given as the weak*-limit of the normalized counting measures of their zeros

$$
\mu_{n}=\frac{1}{n^{\alpha}} \sum\left\{\delta_{z}: Q_{n}(z)=0\right\}
$$

where $\operatorname{deg}\left(Q_{n}\right)=n$ and $\delta_{z}$ is the point mass at $z[5]$. 
For $\alpha=1$, the scaling of the zeros of the polynomial sequence $\left(Q_{n}(x)\right)$ is proportional to their degrees. Inside the unit disk, we found that it is necessary to rescale the zeros according to the power $n^{\alpha}$ to obtain a nonzero distribution.

Remark 3. Both the zero attractor and the asymptotic zero distribution have their advantages and disadvantages in describing the limiting zero set of a sequence of polynomials. The zero attractor gives a complete description of the limiting zero set but gives no information about the distribution of the zeros. In contrast, the asymptotic zero distribution of order $n^{\alpha}$ gives us a description of the zero set that includes the density but its description can be incomplete as it ignores the parts of the zero set which has order $n^{\beta}, 0<\beta<\alpha$.

Before we proceed further, we point out the standard notational conventions in this paper. We continue with the conventions given in [6]. We define $\sqrt[s]{x}$ to be the $s$ root $\exp \left(\frac{\log x}{s}\right)$ with the imaginary part of the logarithm defined on $(-\pi, \pi]$. Next $[x]^{-}$and $\bar{x}$ are defined to be the complex conjugate of $x$.

When we say $g_{n}(x)=O_{V}\left(a_{n}\right)$, where $a_{n}$ a sequence of complex numbers, we mean there exists a constant $C_{V}$ dependent solely on a collection of parameters $V$, so that $\left|g_{n}(x)\right| \leq C_{V}\left|a_{n}\right|$ as $n \rightarrow \infty$. Absence of any $V$ indicates that the constant is uniform. We can make a similar definition for $g_{n}(x)=o_{V}\left(a_{n}\right)$.

We will first compute the zero attractor and the asymptotic zero distribution of $Q_{n}(x)$ but these objects depend on something called a "phase" [6].

Definition 4. Let $\left(L_{k}(x)\right)$ be a sequence of complex valued functions defined on a domain $G$. A set $R(m)$ is called the $m$-th phase (or phase $m$ ) of an " $\left(L_{k}(x)\right)$-Region of Dominance" if and only if

1. If $x \in R(m)$ then for every $k \in \mathbb{N}, k \neq m$ implies $\Re L_{m}(x)>\Re L_{k}(x)$.

2. If an open set $V$ has property (1), $V \subset R(m)$.

Obviously $0 \in Z\left(Q_{n}(x)\right)$ for every $n \in \mathbb{N}$ so we analyze the zeros with the domain $D=\mathbb{D} \backslash\{0\}$ is the open punctured unit disk with

$$
L_{k}(x)=\frac{1}{k} \sqrt[3]{2 L i_{3}\left(x^{k}\right)}, \text { where } L i_{s}(x)=\sum_{n=1}^{\infty} \frac{x^{n}}{n^{s}} .
$$

The functions $L_{k}(x)$ are deeply tied to the asymptotic structure of $Z\left(Q_{n}(x)\right)$ on $D$. In particular, their real part determines the asymptotic location of these zeros on $D$ while their imaginary part plays an important role in determining the asymptotic density of these zeros.

Definition 5. Let $\gamma$ be the level set $\Re L_{1}(x)=\Re L_{2}(x)$ with $\Im x \geq 0$ and $|x| \leq 1$. In [6, Section 4.3], we showed that $\gamma$ can be represented as a curve with a parametrization in polar form as $\gamma(r)=r e^{i \theta(r)}$ where $r \in\left[-x^{*}, 1\right], \theta(r) \in[\pi / 2, \pi]$, and $x^{*}$ is the unique intersection point of $\gamma$ with the negative real axis. 

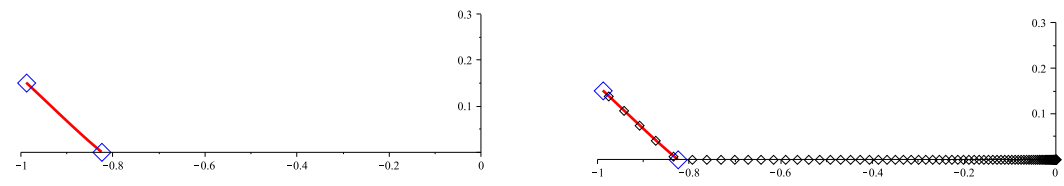

Figure 2: On the left is the level set $\gamma$ (see Definition 5) while on the right is $\gamma$ together with the zeros of $Q_{2200}(x)$ of modulus $<1$ that lie in the second quadrant

In [6] we give an extensive exposition on these phases and prove:

Theorem 6. (Parry-Boyer [6]) Let $D$ be the punctured open unit disk and $L_{k}(x)=$ $\frac{1}{k} \sqrt[3]{2 L i_{3}\left(x^{k}\right)}$. Then there are exactly two nonempty phases $R(1)$ and $R(2)$ with the properties:

1. The boundaries of phases $R(1)$ and $R(2)$ consist of $\gamma$ and its complex conjugate $\bar{\gamma}$; their union equals $\left\{x \in D: \Re L_{1}(x)=\Re L_{2}(x)\right\} \cdot \gamma$ and $\bar{\gamma}$ intersect the real axis at $x^{*} \simeq-0.8250030529$. $\gamma$ intersects the unit circle at $e^{i \theta^{*} \pi}$ where $\theta^{*}$ is the unique solution to $\Re L_{1}\left(e^{i \theta \pi}\right)=\Re L_{2}\left(e^{i \theta \pi}\right)$, for $1 / 2<\theta<1$. Note: $\theta^{*} \simeq 0.9517031251$.

2. $R(2)$ lies in the open left half-plane and

$$
R(2) \cap(-1,1)=\left(-1, x^{*}\right) .
$$

Because we have the proper language, we may now describe the zero attractor and the asymptotic zero distribution.

Theorem 7. The zero attractor $A$ of the sequence of plane partition polynomials $\left(Q_{n}(x)\right)$ is the set

$$
A=\left[x^{*}, 0\right] \cup \gamma \cup \bar{\gamma} \cup S^{1}
$$

where $\gamma$ and $x^{*}$ are given in Definition 5 , and $S^{1}$ is the unit circle.

Theorem 8. The asymptotic zero distribution of order $n$ of the sequence $\left(Q_{n}(x)\right)$ is the normalized Lebesgue measure supported on the unit circle $S^{1}$.

Now we address our second question. Obviously zeros accumulate only along the zero attractor of $\left(Q_{n}(x)\right)$ so we only need to estimate distributions in the four regions given above. By proving Theorem 8 , we will have already attained this estimate about the unit circle.

Theorem 9. Let $-\pi<\theta_{1}<\theta_{2} \leq \pi$, then for every $\epsilon>0$ sufficiently small

$$
\lim _{n \rightarrow \infty} \frac{1}{n}\left|\left\{z \in \mathbb{C}: \arg z \in\left[\theta_{1}, \theta_{2}\right],|z| \in(1-\epsilon, 1+\epsilon), Q_{n}(z)=0\right\}\right|=\frac{1}{2 \pi}\left(\theta_{2}-\theta_{1}\right) .
$$



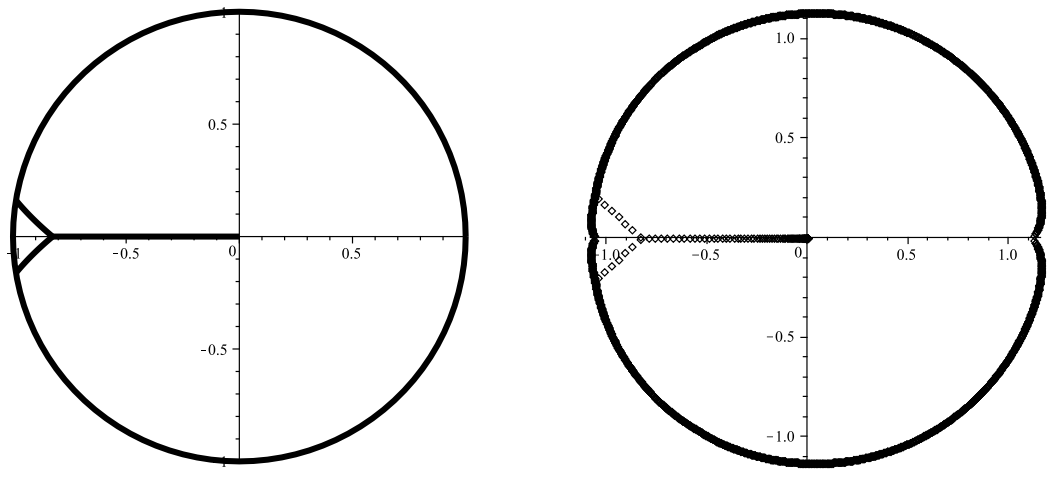

Figure 3: On the left is the zero attractor of $Q_{n}(x)$ while on the right is the zero set of $Q_{2200}(x)$.

So we will focus our attention on the parts of the zero attractor that do not have scale $n$. We will start with the density of $\left[x^{*}, 0\right]$.

Theorem 10. Let $x^{*}<x<y<0$ and $\epsilon>0$, then

$$
\lim _{n \rightarrow \infty} \frac{1}{n^{\frac{2}{3}}}\left|\left\{z \in \mathbb{C}: \Re z \in(x, y),|\Im z|<\epsilon, \quad Q_{n}(z)=0\right\}\right|=\frac{3}{2 \pi} \Im\left[L_{1}(x)-L_{1}(y)\right] .
$$

Then we compute similar theorems for $\gamma$ and $\bar{\gamma}$. Since $Q_{n}(x)$ has real coefficients, its zero set is symmetric about the real axis and so $\gamma$ and its complex conjugate have identical asymptotic distributions. We only then need to prove a theorem for $\gamma$.

Theorem 11. (a) Let $\Psi(z)=3 i\left(L_{2}(z)-L_{1}(z)\right) / 2$. Then $\Psi$ maps the level set $\gamma$ conformally to the line segment $E=\left[\Psi\left(x^{*}\right), \Psi\left(e^{i \pi \theta^{*}}\right)\right]$ where $\Psi\left(x^{*}\right) \simeq 1.4907011097$ and $\Psi\left(e^{i \pi \theta^{*}}\right) \simeq 1.6627531735$.

(b) For every $(\alpha, \beta) \subset E$ and positive sufficiently small $\epsilon_{0}$

$$
\lim _{n \rightarrow \infty} \frac{1}{n^{\frac{2}{3}}}\left|\left\{z \in \mathbb{C}: \Re \Psi(z) \in(\alpha, \beta),|\Im \Psi(z)| \leq \epsilon_{0}, Q_{n}(z)=0\right\}\right|=\frac{\beta-\alpha}{2 \pi} .
$$

\section{The Zero Attractor of Plane Partition Polynomials $\left(Q_{n}(x)\right)$}

When viewing the zero attractor $A$ of $Q_{n}(x)$ it is useful to understand the following definitions [14, page 104]:

$\lim \inf Z\left(Q_{n}(x)\right)=\left\{z \in \mathbb{C}: \forall \epsilon>0, B(z, \epsilon) \cap Z\left(Q_{n}(x)\right) \neq \emptyset\right.$ for all but finitely many $\left.n\right\}$ $\lim \sup Z\left(Q_{n}(x)\right)=\left\{z \in \mathbb{C}: \forall \epsilon>0, B(z, \epsilon) \cap Z\left(Q_{n}(x)\right) \neq \emptyset\right.$ for infinitely many $\left.n\right\}$

One should also note

$$
\lim \inf Z\left(Q_{n}(x)\right) \subseteq A \subseteq \lim \sup Z\left(Q_{n}(x)\right)
$$




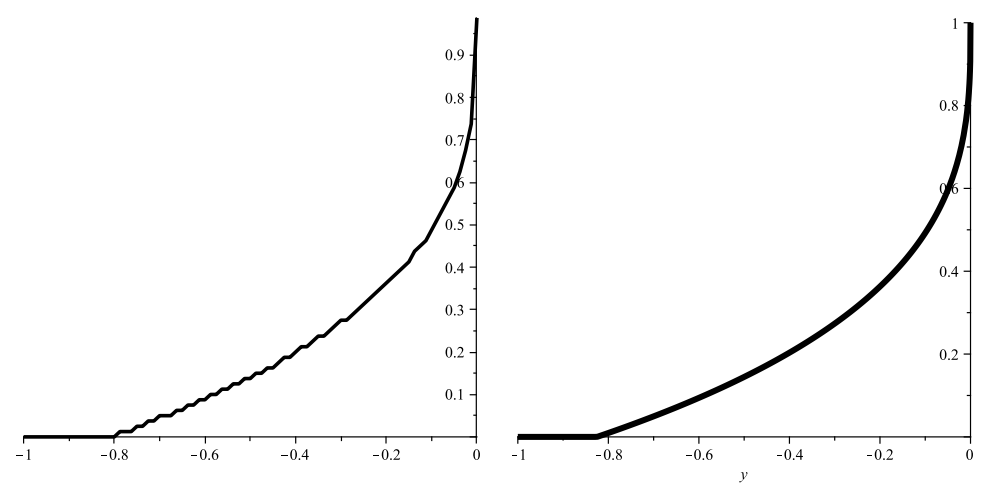

Figure 4: On the left is the cumulative density plot of the zeros $Q_{2200}(x)$ on the negative real axis. On the right is the cumulative distribution predicted by Theorem 10 . The support of the distribution is $\left[x^{*}, 0\right]$.

and when set equality holds

$$
\liminf Z\left(Q_{n}(x)\right)=\lim \sup Z\left(Q_{n}(x)\right)=A .
$$

We will therefore prove Theorem 7 in three steps:

Step 1: Show that “ $\infty " \notin \lim \sup Z\left(Q_{n}(x)\right)$.

Step 2: $\left[x^{*}, 0\right] \cup \gamma \cup \bar{\gamma} \cup S^{1} \subseteq \liminf Z\left(Q_{n}(x)\right)$.

Step 3: $\left(\left[x^{*}, 0\right] \cup \gamma \cup \bar{\gamma} \cup S^{1}\right)^{c} \subseteq \lim \sup Z\left(Q_{n}(x)\right)^{c}$.

Together these three steps imply that

$$
\left[x^{*}, 0\right] \cup \gamma \cup \bar{\gamma} \cup S^{1} \subseteq \liminf Z\left(Q_{n}(x)\right) \subseteq \lim \sup Z\left(Q_{n}(x)\right) \subseteq\left[x^{*}, 0\right] \cup \gamma \cup \bar{\gamma} \cup S^{1},
$$

which determines the zero attractor $A$ since the limit inf and limit sup of the sequence of sets $Z\left(Q_{n}(x)\right)$ agree:

$$
\lim \inf Z\left(Q_{n}(x)\right)=\lim \sup Z\left(Q_{n}(x)\right)=\left[x^{*}, 0\right] \cup \gamma \cup \bar{\gamma} \cup S^{1}=A .
$$

\subsection{Proof of Step 1}

As a technical point, in order to consider the zero attractor in the topology of the one point compactification of $\mathbb{C}$ we must show that " $\infty$ " cannot be part of the zero attractor. To do this it is sufficient to show that " $\infty$ " $\notin \lim \sup Z\left(Q_{n}(x)\right)$ or that there is a compact set $\overline{B\left(0, r_{0}\right)}$ for some $r_{0}>0$ and $N>0$ so that for $n>N Z\left(Q_{n}(x)\right)$ is contained in $\overline{B\left(0, r_{0}\right)}$. 
Lemma 12. For all $r_{0}>1$ there exists a $N>0$ so that for $n>N$

$$
Z\left(Q_{n}(x)\right) \subset B\left(0, r_{0}\right) \subset \overline{B\left(0, r_{0}\right)} .
$$

Therefore " $\infty$ " $\notin A$.

Proof. This is a natural consequence of the asymptotic estimate of $Q_{n}(x)$ outside the unit disk given in [7]. For every $|x|>r_{0}>1$ and positive $\eta$,

$$
Q_{n}(x)=x^{n} \prod_{k=1}^{\infty} \frac{1}{\left(1-x^{-k}\right)^{k+1}}+O_{r_{0}, \eta}\left(x^{n\left(\frac{1}{2}+\eta\right)}\right) .
$$

Now choose any $\eta>0$ and $r_{0}>1$ so that $0 \notin Z\left(Q_{n}(x)\right) \cap B\left(0, r_{0}\right)^{c}$. Then we have

$$
Z\left(\frac{Q_{n}(x)}{x^{n}}\right) \cap B\left(0, r_{0}\right)^{c}=Z\left(Q_{n}(x)\right) \cap B\left(0, r_{0}\right)^{c} .
$$

By equation 2, we find that

$$
\lim _{n \rightarrow \infty} \frac{Q_{n}(x)}{x^{n}}=\prod_{k=1}^{\infty} \frac{1}{\left(1-x^{-k}\right)^{k+1}}
$$

uniformly. Thus, for $0<\epsilon<1$ there exists a $N>0$ so that for $n>N$

$$
\left|\frac{Q_{n}(x)}{x^{n}}\right| \geq \prod_{k=1}^{\infty} \frac{1}{\left(1+|x|^{-k}\right)^{k+1}}-\epsilon>0 .
$$

Hence we see that $Z\left(Q_{n}(x) / x^{n}\right) \cap B\left(0, r_{0}\right)^{c}=\emptyset$ and conclude

$$
Z\left(Q_{n}(x)\right) \cap B\left(0, r_{0}\right)^{c}=\emptyset .
$$

\subsection{Proof of Step 2}

The set liminf $Z\left(Q_{n}(x)\right)$ is intrinsically tied to the asymptotics of $Q_{n}(x)$ in the complex plane. This connection is given by the following theorem [15].

Theorem 13. (Sokal) Let $G$ be a domain in $\mathbb{C}$ with $x_{0} \in G$. Let $\left(Q_{n}(x)\right)$ be a sequence of analytic functions on $G$, and let $\left(a_{n}\right)$ be a sequence of positive real constants such that $\left(\left|Q_{n}(x)\right|^{a_{n}}\right)$ are uniformly bounded on compact subsets of $G$. Suppose that there does not exist a neighborhood $U$ containing $x_{0}$ and a function $v$ on $U$ that is either harmonic or else identically $-\infty$ such that

$$
\liminf \log \left|Q_{n}(x)\right|^{a_{n}} \leq v(x) \leq \lim \sup \log \left|Q_{n}(x)\right|^{a_{n}} .
$$

Then $x_{0} \in \liminf Z\left(Q_{n}(x)\right)$. 
To make more clear this connection if $\left|Q_{n}(x)\right| \sim v(x)^{\frac{1}{a_{n}}}$ then

$$
v(x)=\lim _{n \rightarrow \infty} a_{n} \ln \left|Q_{n}(x)\right| .
$$

Thus where asymptotic approximations in a sense "fail to be harmonic" is $\lim \inf Z\left(Q_{n}(x)\right)$. In [7] we have computed the asymptotics of $Q_{n}(x)$. They are given by equation 2 and the following two theorems.

Theorem 14. (a) Let $X \subset R(1) \backslash\left[x^{*}, 0\right]$ be a compact set and $x \in X$, then

$$
Q_{n}(x)=\sqrt[12]{1-x} \sqrt{\frac{L_{1}(x)}{6 \pi n^{\frac{4}{3}}}} \exp \left(n^{\frac{2}{3}}\left(\frac{3}{2} L_{1}(x)\right)\right)\left(1+O_{X}\left(\frac{1}{n^{\frac{1}{3}}}\right)\right) .
$$

(b) Let $X \subset R(2)$ be a compact and let $x \in X$, then

$$
Q_{n}(x)=(-1) \sqrt[n]{\sqrt[24]{1-x^{2}}} \sqrt[8]{\frac{1-x}{1+x} \sqrt{\frac{L_{2}(x)}{6 \pi n^{\frac{4}{3}}}}} \exp \left(n^{\frac{2}{3}}\left(\frac{3}{2} L_{2}(x)\right)\right)\left(1+O_{X}\left(\frac{1}{n^{\frac{1}{3}}}\right)\right)
$$

Theorem 15. (a) Let $X \subset\left(x^{*}, 0\right)$ be a compact set and let $x \in X$, we have that

$$
\begin{aligned}
Q_{n}(x) & =\sqrt[12]{1-x}\left[\sqrt{\frac{L_{1}(x)}{6 \pi n^{\frac{4}{3}}}} \exp \left(n^{\frac{2}{3}}\left(\frac{3}{2} L_{1}(x)\right)\right)\right]^{-}\left(1+O_{X}\left(\frac{1}{n^{\frac{1}{3}}}\right)\right) \\
& +\sqrt[12]{1-x} \sqrt{\frac{L_{1}(x)}{6 \pi n^{\frac{4}{3}}}} \exp \left(n^{\frac{2}{3}}\left(\frac{3}{2} L_{1}(x)\right)\right)\left(1+O_{X}\left(\frac{1}{n^{\frac{1}{3}}}\right)\right) .
\end{aligned}
$$

(b) Let $X \subset\left\{x: \Re L_{1}(x)=\Re L_{2}(x)\right\} \backslash\left\{x^{*}\right\}$ be a compact set and let $x \in X$, then

$$
\begin{aligned}
Q_{n}(x) & =\sqrt[12]{1-x} \sqrt{\frac{L_{1}(x)}{6 \pi n^{\frac{4}{3}}}} \exp \left(n^{\frac{2}{3}}\left(\frac{3}{2} L_{1}(x)\right)\right)\left(1+O_{X}\left(\frac{1}{n^{\frac{1}{3}}}\right)\right) \\
& +(-1) \sqrt[n]{1-x^{2}} \sqrt[8]{\frac{1-x}{1+x}} \sqrt{\frac{L_{2}(x)}{6 \pi n^{\frac{4}{3}}}} \exp \left(n^{\frac{2}{3}}\left(\frac{3}{2} L_{2}(x)\right)\right)\left(1+O_{X}\left(\frac{1}{n^{\frac{1}{3}}}\right)\right) .
\end{aligned}
$$

So we will apply Theorem 13 in two settings. The first would be with $a_{n}=1 / n$ and $G=\mathbb{C}$ and then we will let $a_{n}=1 / n^{\frac{2}{3}}$ and $G=\mathbb{D} \backslash\{0\}$.

\subsubsection{Application of Theorem 13 with $a_{n}=1 / n$ and $G=\mathbb{C}$}

Since any compact subset of $X \subset \mathbb{C}$ is bounded, one can observe that

$$
\left|Q_{n}(x)\right|^{\frac{1}{n}} \leq Q_{n}(|x|)^{\frac{1}{n}} \leq \sup _{x \in X} Q_{n}(|x|)^{\frac{1}{n}}
$$

Using Theorems 14 and 15 as well as equation 2, we observe the following limit: 
Corollary 16. The limits below hold uniformly on compact subsets of the region:

$$
\lim _{n \rightarrow \infty} \frac{1}{n} \ln \left|Q_{n}(x)\right|= \begin{cases}\ln |x|, & |x| \geq 1 \\ 0, & |x|<1\end{cases}
$$

The existence of these limits imply that $\sup _{x \in X} Q_{n}(|x|)^{\frac{1}{n}}$ is bounded. Thus $\left|Q_{n}(x)\right|^{\frac{1}{n}}$ is uniformly bounded. We then observe that if $v(x)$ in Theorem 13 was harmonic on the unit circle, then

$$
v(x)=\lim _{n \rightarrow \infty} \frac{1}{n} \ln \left|Q_{n}(x)\right|= \begin{cases}\ln |x|, & |x| \geq 1 \\ 0, & |x|<1 .\end{cases}
$$

The function $v(x)$ cannot be differentiable on the unit circle; in particular, it cannot be harmonic. So Theorem 13 applies and gives

Lemma 17. $S^{1} \subset \liminf Z\left(Q_{n}(x)\right)$.

\subsubsection{Application of Theorem 13 with $a_{n}=1 / n^{\frac{2}{3}}$ and $G=\mathbb{D} \backslash\{0\}$}

We will observe that

$$
\left|Q_{n}(x)\right| \leq Q_{n}(1)=P L(n) .
$$

By using E.M. Wright's approximation for PL(n) [17]

$$
P L(n) \sim \frac{\zeta(3)^{\frac{7}{36}}}{2^{\frac{11}{36}} \pi^{\frac{1}{2}} n^{\frac{25}{36}}} \exp \left(\frac{3}{2} n^{\frac{2}{3}} \sqrt[3]{2 \zeta(3)}+2 \int_{0}^{\infty} \frac{y \ln y}{e^{2 \pi y}-1} d y\right)
$$

it is clear that $\left|Q_{n}(x)\right|^{1 / n^{\frac{2}{3}}}$ is bounded uniformly on all subsets of the open unit disk. Theorems 14 and 15 imply

Corollary 18. The limits below hold uniformly on compact subsets of the region:

$$
\lim _{n \rightarrow \infty} \frac{1}{n^{\frac{2}{3}}} \ln \left|Q_{n}(x)\right|= \begin{cases}\frac{3}{2} \Re L_{1}(x), & x \in R(1), \\ \frac{3}{2} \Re L_{2}(x), & x \in R(2), \\ \frac{3}{2} \Re L_{2}(x)=\frac{3}{2} \Re L_{1}(x), \quad x \in \gamma \cup \bar{\gamma}\end{cases}
$$

We then observe that if $v(x)$ in Theorem 13 was harmonic then

$$
v(x)=\lim _{n \rightarrow \infty} \frac{1}{n^{\frac{2}{3}}} \ln \left|Q_{n}(x)\right|= \begin{cases}\frac{3}{2} \Re L_{1}(x), & x \in R(1), \\ \frac{3}{2} \Re L_{2}(x), & x \in R(2), \\ \frac{3}{2} \Re L_{2}(x)=\frac{3}{2} \Re L_{1}(x), \quad x \in \gamma \cup \bar{\gamma} .\end{cases}
$$

So if $v(x)$ is harmonic in a neighborhood of $\gamma \cup \bar{\gamma}$ then, by principle of analytic continuation, $\Re L_{1}(x)=\Re L_{2}(x)$ on $\mathbb{D} \backslash\{0\}$. This is simply untrue as $L_{1}(x)$ and $L_{2}(x)$ are distinct. Therefore we have the set containment for the level sets $\gamma$ and $\bar{\gamma}$ : 
Lemma 19. $\gamma \cup \bar{\gamma} \subset \liminf Z\left(Q_{n}(x)\right)$.

In [6] we have noted that $\Re L_{k}(x)$ is not harmonic on $\left\{x: x^{k} \leq 0\right\}$. Therefore $v(x)$ cannot be harmonic on $R(1) \cap\{x: x \leq 0\}$ and $R(2) \cap\left\{x: x^{2} \leq 0\right\}$. By Theorem 6 $R(1) \cap\{x: x \leq 0\}$ is the interval $\left(x^{*}, 0\right)$ while $R(2) \cap\left\{x: x^{2} \leq 0\right\}$ is empty. Theorem 13 applies again to give us:

Lemma 20. $\left[x^{*}, 0\right] \subset \liminf Z\left(Q_{n}(x)\right)$.

Combining Lemmas 17, 19, and 20, we now obtain

Lemma 21. $\left[x^{*}, 0\right] \cup \gamma \cup \bar{\gamma} \cup S^{1} \subset \liminf Z\left(Q_{n}(x)\right)$.

\subsection{Proof of Step 3}

Recall that $x \notin \lim \sup Z\left(Q_{n}(x)\right)$ if and only if there exists a $\epsilon, N>0$ so that for $n>N$, $B(x, \epsilon)$ is disjoint from $Z\left(Q_{n}(x)\right)$. Like $\lim \inf Z\left(Q_{n}(x)\right), \lim \sup Z\left(Q_{n}(x)\right)^{c}$ is tied to the asymptotic approximations of $Q_{n}(x)$ in the complex plane. This connection is given by an application of Hurwitz's Theorem: Let $G \subset \mathbb{C}$ be a region and suppose the sequence of holomorphic functions $f_{n}(x)$ converges uniformly on compact subsets of $G$ to $f(x)$. If $f(x)$ is not identically zero, $B\left(x_{0}, r\right) \subset G$ and $f(x) \neq 0$ on the boundary of our open ball, then there exists an $N$ such that for all $n>N f(x)$ and $f_{n}(x)$ have the same number of zeros in $B\left(x_{0}, r\right)$ ([9, Page 152]).

Theorem 22. Let $G \subset \mathbb{C}$ be a region. Suppose there exists a sequence of holomorphic functions $\left(g_{n}(x)\right)$ such that

$$
\lim _{n \rightarrow \infty} Q_{n}(x) / g_{n}(x)=1
$$

uniformly on compact subsets of $G$. If $g_{n}\left(z_{0}\right)$ is not zero on some neighborhood of $z_{0}$ inside $G$, then $z_{0} \notin \limsup Z\left(Q_{n}(x)\right)$.

Proof. We begin by choosing any nonempty open subset of $G$ so that $g_{n}(x)$ is nonzero on the closure of this neighborhood. It is then the case that $Q_{n}(x) / g_{n}(x)$ is a sequence of holomorphic functions which converge uniformly to 1 on the closure of this neighborhood. Then Hurwitz's Theorem applies showing that for all but finitely many $n Q_{n}(x)$ is nonzero in $B\left(x_{0}, r\right)$. Thus, by definition $x_{0} \notin \lim \sup Z\left(Q_{n}(x)\right)$.

Lemma 23. $\overline{\mathbb{D}}^{c} \subset \lim \sup Z\left(Q_{n}(x)\right)^{c}$.

Proof. If $|x|>1$, then

$$
g_{n}(x)=x^{n} \prod_{k=1}^{\infty} \frac{1}{\left(1-x^{-k}\right)^{k+1}}
$$

is nonzero outside the closed unit disk. By equation 2, it follows that the polynomials $Q_{n}(x)$ themselves are nonzero there as well.

Lemma 24. $\overline{\mathbb{D}} \backslash\left(S^{1} \cup\left[x^{*}, 0\right] \cup \gamma \cup \bar{\gamma}\right) \subset \lim \sup Z\left(Q_{n}(x)\right)^{c}$. 
Proof. By Theorem 6 the closed unit disk can be decomposed into a disjoint union

$$
\overline{\mathbb{D}}=S^{1} \cup\left[x^{*}, 0\right] \cup \gamma \cup \bar{\gamma} \cup R(1) \backslash\left[x^{*}, 0\right] \cup R(2) .
$$

In other words, we have the set equality

$$
\overline{\mathbb{D}} \backslash\left(S^{1} \cup\left[x^{*}, 0\right] \cup \gamma \cup \bar{\gamma}\right)=R(1) \backslash\left[x^{*}, 0\right] \cup R(2) .
$$

Theorem 14 now allows us to say that if $x \in R(1) \backslash\left[x^{*}, 0\right]$ then

$$
g_{n}(x)=\sqrt[12]{1-x} \sqrt{\frac{L_{1}(x)}{n^{\frac{4}{3}} 6 \pi}} \exp \left(n^{\frac{2}{3}}\left(\frac{3}{2} L_{1}(x)\right)\right) \neq 0 .
$$

If $x \in R(2)$, then

$$
g_{n}(x)=(-1) \sqrt[n]{\sqrt[24]{1-x^{2}}} \sqrt[8]{\frac{1-x}{1+x}} \sqrt{\frac{L_{2}(x)}{n^{\frac{4}{3}} 6 \pi}} \exp \left(n^{\frac{2}{3}}\left(\frac{3}{2} L_{2}(x)\right)\right) \neq 0 .
$$

Lemma 25.

$$
\begin{aligned}
& \left(S^{1} \cup\left[x^{*}, 0\right] \cup \gamma \cup \bar{\gamma}\right)^{c} \\
& =\left[\overline{\mathbb{D}} \backslash\left(S^{1} \cup\left[x^{*}, 0\right] \cup \gamma \cup \bar{\gamma}\right)\right] \cup \overline{\mathbb{D}}^{c} \subset \limsup Z\left(Q_{n}(x)\right)^{c} .
\end{aligned}
$$

So we conclude the proof as we said we would do in the introduction; that is, by Lemmas 21 and 25,

$$
S^{1} \cup\left[x^{*}, 0\right] \cup \gamma \cup \bar{\gamma} \subseteq \liminf Z\left(Q_{n}(x)\right) \subseteq A \subseteq \lim \sup Z\left(Q_{n}(x)\right) \subseteq S^{1} \cup\left[x^{*}, 0\right] \cup \gamma \cup \bar{\gamma},
$$

and so the zero attractor $A$ is given by

$$
A=S^{1} \cup\left[x^{*}, 0\right] \cup \gamma \cup \bar{\gamma} .
$$

\section{Asymptotic Zero Distribution of Order $n$}

We now have found the zero attractor $A$ so the next problem is to find how the zeros are distributed among the different curves of $A$.

In this section, we work with normalized counting measure of the zeros of $Q_{n}(x)$ of order $n$. For simplicity of notation, we will exclude contribution of 0 which is a simple zero for all the polynomials $Q_{n}(x)$. So we will use $\mu_{n}$ given as

$$
\mu_{n}=\frac{1}{n} \sum\left\{\delta_{x}: Q_{n}(x)=0, x \neq 0\right\} .
$$


Theorem 26. The normalized counting measures $\mu_{n}$ converge weakly to normalized Lebesgue measure $\mu$ on the unit circle.

Proof. We will use the characterization of weak convergence in [1, Theorem 2.2, page 14]: Let $\mathcal{U}$ be a collection of open subsets of $\mathbb{C}$ such that (i) $\mathcal{U}$ is closed under finite intersections and (ii) each open set is either a finite or countable union of elements of $\mathcal{U}$. If $\mu_{n}(U) \rightarrow \mu(U)$ for every $U$ in $\mathcal{U}$, then $\mu_{n}$ converges weakly to $\mu$.

We will use the collection of open subsets of $\mathbb{C}$ consisting of annular wedges

$$
W\left(r_{1}, r_{2} ; \phi_{1}, \phi_{2}\right)=\left\{x \in \mathbb{C}: r_{1}<|x|<r_{2}, \phi_{1}<\arg x<\phi_{2}\right\}
$$

where $r_{1}, r_{2}, \phi_{1}, \phi_{2} \in \mathbb{Q}^{+}$with $r_{1}, r_{2} \neq 1, r_{1}<r_{2}$, and $\phi_{2}-\phi_{1}<\pi$.

Since the zero attractor $A$ must lie inside the closed unit disk, it is easy to verify that

$$
\limsup _{n \rightarrow \infty} \mu_{n}\left(W\left(r_{1}, r_{2} ; \phi_{1}, \phi_{2}\right)\right)=0, \quad r_{1}>1 .
$$

For the reminder of the proof it is convenient to work with a normalized family of polynomials $P_{n}(x)=Q_{n}(x) / x$ so $P_{n}(x)$ have exactly the same nonzero roots as $Q_{n}(x)$ as well as $P_{n}(0)=1, \operatorname{deg}\left(P_{n}\right)=n-1$, and the coefficient $\left[x^{n-1}\right] P_{n}(x)=1$.

Let $\epsilon>0$ be given. Consider $W\left(r_{1}, r_{2} ; \phi_{1}, \phi_{2}\right)$ with $r_{2} \leq 1-\epsilon$. Recall Jensen's formula from [9, pages 280-281]: suppose that $f(x)$ is an analytic function on an open neighborhood $B\left(x_{0}, r\right)$, let $a_{0}, a_{1}, a_{2}, \ldots, a_{m}$ be the zeros inside this neighborhood. Then

$$
\ln \left|f\left(x_{0}\right)\right|=\sum_{k=0}^{m} \ln \frac{\left|a_{k}\right|}{r}+\frac{1}{2 \pi} \int_{0}^{2 \pi} \ln \left|f\left(x_{0}+r e^{i t}\right)\right| d t .
$$

For the sake of completeness, we sketch how Jensen's formula is used to count zeros for $P_{n}(x)$ with $r=1$. We have the bounds

$$
\begin{aligned}
\frac{1}{2 \pi} \int_{0}^{2 \pi} \ln \left|P_{n}\left(e^{i t}\right)\right| d t & =\ln \left|P_{n}(0)\right|+\sum\left\{-\ln |z|: P_{n}(z)=0,|z|<1\right\} \\
& \geq-\sum\left\{\ln |z|: P_{n}(z)=0,|z|<1-\epsilon\right\}>\epsilon n \mu_{n}(B(0,1-\epsilon) .
\end{aligned}
$$

On the other hand, we have the easy bound $\max \left\{\left|P_{n}(z)\right|:|z|=1\right\} \leq P_{n}(1)=P L(n)$. Hence, we have the estimate

$$
\mu_{n}(B(0,1-\epsilon)) \leq \frac{1}{2 \pi \epsilon n} \ln P L(n)
$$

Since by Wright's result $P L(n)$ has subexponential growth, we conclude that

$$
\limsup _{n \rightarrow \infty} \mu_{n}(B(0,1-\epsilon))=0 .
$$

This implies, of course, that $\limsup _{n \rightarrow \infty} \mu_{n}\left(W\left(r_{1}, r_{2} ; \phi_{1}, \phi_{2}\right)\right)=0$ when $r_{2}<1$.

For the final case we let $0<r_{1}<1<r_{2}$. We need a theorem of Erdös and Turán [11]. Let $P(x)$ be a polynomial $P(x)=a_{0}+a_{1} x+\cdots+a_{d} x^{d}$ where $a_{0} a_{d} \neq 0$. Set 
$M=\sum\left|a_{j}\right| / \sqrt{\left|a_{0} a_{d}\right|}$ and set $N(\alpha, \beta)$ to be the number of zeros of $P(x)$ whose arguments lie in the interval $[\alpha, \beta]$. Then

$$
\left|\frac{N(\alpha, \beta)}{d}-\frac{\beta-\alpha}{2 \pi}\right|<16 \frac{\sqrt{\ln M}}{\sqrt{d}} .
$$

For the polynomials $P_{n}(x)$, we find

$$
\left|\mu_{n+1}\left(W\left(r_{1}, r_{2} ; \phi_{1}, \phi_{2}\right)\right)-\frac{\phi_{2}-\phi_{1}}{2 \pi}\right|<16 \frac{\sqrt{\ln [P L(n+1)]}}{\sqrt{n}} .
$$

Since the plane partition numbers $P L(n)$ have subexponential growth, we must have

$$
\lim _{n \rightarrow \infty} \mu_{n}\left(W\left(r_{1}, r_{2} ; \phi_{1}, \phi_{2}\right)\right)=\frac{\phi_{2}-\phi_{1}}{2 \pi} .
$$

By the characterization of weak convergence of probability measures, the normalized counting measures do indeed converge to normalized Lebesgue measure on the unit circle.

\section{Asymptotic Zero Distributions of Order $n^{2 / 3}$}

The zero attractor $A$ of $Q_{n}(x)$ consists of the unit circle together with $\left[x^{*}, 0\right] \cup \gamma \cup \bar{\gamma}$. In the above section, we showed that the zeros in the limit are uniformly distributed along the unit circle and are of order $O(n)$. In this section, we determine the asymptotic zero distributions along the level set $\gamma$ and the line segment $\left[x^{*}, 0\right]$ along the negative real axis both of which are of order $n^{2 / 3}$.

\subsection{Distribution of Zeros along the level sets $\gamma$ and $\bar{\gamma}$ (Proof of Theorem 11)}

We will apply the Density Theorem from [12]; see [5] for another exposition. Recall the setup of this theorem. Let $\psi$ be a conformal mapping from a neighborhood $U$ of an analytic arc $C$ to a neighborhood of the unit circle of the form $W=W\left(r_{1}, r_{2} ; \alpha, \beta\right)$ (see (3) for this notation), where $r_{1}<1<r_{2}$ and $C$ is mapped to the circular arc $\Gamma$ : $e^{i t}, \alpha \leq t \leq \beta$. Let $W_{+}=W\left(1, r_{2} ; \alpha, \beta\right)$ and $W_{-}=W\left(r_{1}, 1 ; \alpha, \beta\right)$ so $U=\psi^{-1}\left(W_{+}\right) \cup C \cup \psi^{-1}\left(W_{-}\right)$.

Theorem 27. [12, Section 6] If $\left(T_{n}(x)\right)$ is a sequence of analytic functions on $U$ such that

$$
T_{n}(x)=1+a_{n}(x) \psi^{c_{n}}(x)+e_{n}(x)
$$

where $\left(c_{n}\right)$ is an unbounded increasing sequence of positive numbers and $\left(a_{n}(x)\right)$ is a sequence of analytic functions that satisfy uniformly on $U$ as $n \rightarrow \infty:\left|a_{n}(x)\right| \geq \delta>0$, $\frac{a_{n}^{\prime}(x)}{n a_{n}(x)}=o(1)$, and $e_{n}(x)=o_{X}\left(a_{n}(x) \psi^{c_{n}}(x)\right)$ ( $X$ is a compact subset of $W$ ) and further

$$
e_{n}(x)= \begin{cases}o_{X}\left(\psi(x)^{c_{n}}\right), & x \in \psi^{-1}\left(W_{+}\right) \\ o_{X}(1), & x \in \psi^{-1}\left(W_{-}\right)\end{cases}
$$

THE ELECTRONIC JOURNAL OF COMBINATORICS 18(2) (2012), \#P30 
where $X$ is a compact subset of $W_{ \pm}$. Then for any $\epsilon>0$ all the zeros of $T_{n}(x)$ lie in $C_{\epsilon}$ for $n$ sufficiently large, where $C_{\epsilon}$ is the $\epsilon$-neighborhood of the analytic arc $C$ and

$$
\lim _{n \rightarrow \infty} \frac{1}{c_{n}} \sum\left\{\delta_{\psi(z)}: z \in Z\left(T_{n}\right) \cap C_{\epsilon}\right\} \rightarrow \mu
$$

where $\mu$ is normalized Lebesgue measure on the unit circle restricted to the circular arc $\psi(C)$. In particular, the zero density measure $\nu_{Z}$ for the family $\left(T_{n}(x)\right)$ is $\nu_{Z}=\mu \circ \psi$ on the $\operatorname{arc} C$.

To apply this density theorem, we require an alternate form of Theorems 14 and 15 which is given in Remark 27 in [7]; if $X$ is compact and disjoint from negative and imaginary axes, then we have

$$
\begin{aligned}
Q_{n}(x) & =\sqrt[12]{1-x} I_{1,1, n}(x)+(-1) \sqrt[n]{1-x^{2}} \sqrt[8]{\frac{1-x}{1+x}} I_{1,2, n}(x), \text { where } \\
I_{h, k, n}(x) & =\sqrt{\frac{L_{k}(x)}{n^{\frac{4}{3}} 6 \pi}} \exp \left(n^{\frac{2}{3}}\left(\frac{3}{2} L_{k}(x)\right)\right)\left(1+O_{X}\left(\frac{1}{n^{\frac{1}{3}}}\right)\right) .
\end{aligned}
$$

We define $T_{n}(x)$ by the normalization

$$
T_{n}(x)=\frac{Q_{n}(x)}{\sqrt[12]{1-x} I_{1,1, n}(x)}
$$

Observe that if $x \neq 0,1$, then $I_{h, k, n}(x)$ does not vanish and so $Z\left(Q_{n}(x)\right)$ and $Z\left(T_{n}(x)\right)$ differ by at most two zeros. For the plane partition polynomials, the required functions for the density result are

$$
\begin{aligned}
T_{n}(x) & =1+a_{n}(x) \psi(x)^{n^{2 / 3}}+e_{n}(x), \quad c_{n}=n^{2 / 3} \\
\psi(x) & =e^{\frac{3}{2}\left(L_{2}(x)-L_{1}(x)\right)} \\
a_{n}(x) & =(-1)^{n} \frac{\sqrt[24]{1-x^{2}}}{\sqrt[12]{1-x}} \sqrt[8]{\frac{1-x}{1+x}} \sqrt{\frac{L_{2}(x)}{L_{1}(x)}} \\
e_{n}(x) & =O_{X}\left(\frac{1}{n^{1 / 3}} a_{n}(x) \psi(x)^{n^{2 / 3}}\right)= \begin{cases}o_{X}\left(\psi(x)^{c_{n}}\right), & x \in R(2), \\
o_{X}(1), & x \in R(1) .\end{cases}
\end{aligned}
$$

For convenience of the reader, we give a brief indication that the conditions of the theorem are met. It is easy to see that $\psi^{-1}\left(W_{-}\right) \subset R(1)$ and $\psi^{-1}\left(W_{+}\right) \subset R(2)$; that is, if $x \in R(1)$, then $\Re L_{1}(x)>\Re L_{2}(x)$ by its definition so

$$
|\psi(x)|=\exp \left(\frac{3}{2} \Re\left(L_{2}(x)-L_{1}(x)\right)\right)<1
$$

and, in addition,

$$
e_{n}(x)=O_{X}\left(\frac{1}{n^{1 / 3}} a_{n}(x) \psi(x)^{n^{2 / 3}}\right)=o_{X}(1)
$$


Similarly, if $x \in R(2)$ then $\Re L_{2}(x)>\Re L_{1}(x)$ or $|\psi(x)|>1$, so

$$
e_{n}(x)=O_{X}\left(\frac{1}{n^{1 / 3}} a_{n}(x) \psi(x)^{n^{2 / 3}}\right)=o_{X}\left(\psi(x)^{n^{2 / 3}}\right) .
$$

On $\gamma$, we have by definition $\Re L_{1}(x)=\Re L_{2}(x)$, so

$$
|\psi(x)|=\exp \left(\frac{3}{2} \Re\left(L_{2}(x)-L_{1}(x)\right)\right)=1 .
$$

For $a_{n}(x)$, we first observe that $\left|a_{n}(x)\right|$ is independent of $n$ and so is bounded as $n \rightarrow \infty$. It is bounded away from zero, because $a_{n}(x)$ has roots only when $1-x^{2}$ or when $L_{2}(x)=$ $\sqrt[3]{2 L i_{3}\left(x^{2}\right)}$ vanish; that is, at $x= \pm 1$ and 0 . Proposition 21 in [6] proved these points do not lie on $\gamma$ so we can choose the neighborhood small enough so they are excluded.

To finish the proof of the density result we need to address why $\psi$ is conformal in a neighborhood of $\gamma$. We use a special class of univalent functions called starlike (see the book [10, Chapter 2]).

Definition 28. Let $f(x)$ be an analytic function of $\mathbb{D}$ with $f(0)=0$ and $f^{\prime}(1)=1$. The function $f(x)$ is starlike if and only if $f(\mathbb{D})$ is starlike (convex with respect to the origin).

See [10, Section 2.5] for the following:

Lemma 29. Starlike functions are univalent. An analytic function $f(x)$ on $\mathbb{D}$ with $f(0)=$ 0 and $f^{\prime}(0)=1$ is starlike if and only if

$$
\Re\left(x \frac{f^{\prime}(x)}{f(x)}\right)>0 .
$$

Lemma 30. The function $L_{1}(x)=\sqrt[3]{2 L i_{3}(x)}$ satisfies the inequality

$$
\Re\left(z \frac{\left(x L_{1}^{\prime}(x)\right)^{\prime}}{x L_{1}^{\prime}(x)}\right)=\Re\left(\frac{L i_{1}(x)}{L i_{2}(x)}-\frac{2}{3} \frac{L i_{2}(x)}{L i_{3}(x)}\right)>0, \text { for all } x \in \mathbb{D} .
$$

Because of the computational nature and length of the proof of this lemma, we give its proof in the appendix.

Lemma 31. Let $f(x)=\frac{3}{2}\left(x L_{1}^{\prime}(x)\right)^{3}=\frac{L i_{2}(x)^{3}}{L i_{3}(x)^{2}}$. Then we have (a) $f(x)$ is starlike.

(b) $z L_{1}^{\prime}(z)$ is univalent.

Proof. (a) By cubing $L_{1}(x)$ we eliminate the branch cut which causes $f(x)$ to be indeed analytic. Trivially $f(0)=0$, and $f^{\prime}(0)=1$. Last observe the following trick:

$$
\Re\left(x \frac{f^{\prime}(x)}{f(x)}\right)=\Re\left(x \frac{\left(\left(x L_{1}^{\prime}(x)\right)^{3}\right)^{\prime}}{\left(x L_{1}^{\prime}(x)\right)^{3}}\right)=3 \Re\left(x \frac{\left(x L_{1}^{\prime}(x)\right)^{\prime}}{x L_{1}^{\prime}(x)}\right)>0 .
$$

(b) By part (a), $\frac{3}{2}\left(z L_{1}^{\prime}(z)\right)^{3}$ is univalent. Hence $\left(z L_{1}^{\prime}(z)\right)^{3}$ and $z L_{1}^{\prime}(z)$ are univalent since scaling and the single valued cube root are both univalent. 
With these brief comments about univalent functions, we can continue with our proof of the density result. Our next step appeared in our paper [6] but we restate it because of its importance to the argument.

Theorem 32. If $x \in \mathbb{D}$ and is nonzero, then for every $k \neq j$,

$$
L_{k}^{\prime}(x) \neq L_{j}^{\prime}(x)
$$

Proof. We show the contrapositive. Therefore, we suppose there are distinct positive integers $k, j$ so that $L_{k}^{\prime}(x)=L_{j}^{\prime}(x)$ for some $x \in \mathbb{D} \backslash\{0\}$. Therefore, $x$ also solves the equation $x L_{k}^{\prime}(x)=x L_{j}^{\prime}(x)$. But, $x L_{k}^{\prime}(x)=x^{k} L_{1}^{\prime}\left(x^{k}\right)$. Thus,

$$
x^{k} L_{1}^{\prime}\left(x^{k}\right)=x^{j} L_{1}^{\prime}\left(x^{j}\right) .
$$

Lemma 31 states: $x L_{1}^{\prime}(x)$ is univalent and thus $x^{k}=x^{j}$. Hence $x$ would be either zero or on the unit circle.

Lemma 33. The function $\psi(x)=\exp \left(\frac{3}{2}\left(L_{2}(x)-L_{1}(x)\right)\right)$ is conformal on a neighborhood of $\gamma$.

Proof. It is enough to show that $\psi^{\prime}(x)$ is nonzero on $\gamma$ since $\gamma$ is compact. Note that $\gamma$ can be described also as $|\psi(x)|=1$. Since we have that

$$
\psi^{\prime}(x)=\frac{3}{2}\left(L_{1}^{\prime}(x)-L_{2}^{\prime}(x)\right) \psi(x),
$$

the derivative $\psi^{\prime}(x)=0$ only if $L_{1}^{\prime}(x)=L_{2}^{\prime}(x)$. By Theorem 32, this cannot be the case so the proof is complete.

Now all the hypotheses of the Density Theorem are met so we may conclude that for any $\epsilon_{0}>0$

$$
\lim _{n \rightarrow \infty} \frac{1}{n^{2 / 3}}\left|\left\{z \in \mathbb{C}: \Im \psi(z) \in(\alpha, \beta),|\Re \psi(z)| \leq \epsilon_{0}, Q_{n}(z)=0\right\}\right|=\frac{\beta-\alpha}{2 \pi} .
$$

It seems very natural to use a line segment instead of an arc of the unit circle to describing the density. This is easily done as follows. Define $\Psi(z)=i \ln f(z)=\frac{3 i}{2}\left(L_{2}(z)-\right.$ $\left.L_{1}(z)\right)$. Then the function $\Psi(z)$ maps the set $\psi^{-1}(W)$ to the rectangle given by

$$
\Psi\left(x^{*}\right) \leq \Re \Psi(z) \leq \Psi\left(e^{i \theta^{*} \pi}\right) \text { and }|\Im \Psi(z)| \leq \epsilon_{0}
$$

and $\gamma$ to the line segment $\left[\Psi\left(x^{*}\right), \Psi\left(e^{i \theta^{*} \pi}\right)\right]$ so we now have both parts of Theorem 11 .

\subsection{Distribution of the Zeros of $Q_{n}(x)$ along the negative axis (Proof of Theorem 10)}

In this section, we find the distribution of zeros along the line segment $\left[x^{*}, 0\right]$. The Density Theorem is not directly applicable; however, the proof in [5] can be modified to handle this situation. We begin with a simple lemma. 
Lemma 34. Let

$$
S_{n}=\left\{x \in\left(x^{*}, 0\right): \sqrt{L_{1}(x)} e^{\frac{3}{2} n^{\frac{2}{3}} L_{1}(x)} \in \mathbb{R} \backslash\{0\}\right\} .
$$

then for any $x, y \in\left(x^{*}, 0\right)$ and any $n$ we can choose $x_{n}, y_{n} \subset S_{n}$ so that $x_{n} \rightarrow x$ and $y_{n} \rightarrow y$ as $n \rightarrow \infty$.

This lemma allows us to use the Argument Principle: if $\Gamma_{\epsilon}$ is a simple closed curve then the number of zeros of $Q_{n}(x)$ contained in the interior of $\Gamma_{\epsilon}$ is given by

$$
\frac{1}{2 \pi i} \int_{\Gamma_{\epsilon}} \frac{Q_{n}^{\prime}(z)}{Q_{n}(z)} d z
$$

For $\epsilon>0, n \in \mathbb{N}$, and $x_{n}, y_{n}$ as in Lemma 34 we define $\Gamma_{\epsilon}=\Gamma_{+}-\Gamma_{-}+\Gamma_{x_{n}}-\Gamma_{y_{n}}$.

$$
\begin{aligned}
& \Gamma_{+}=\left\{t+i \epsilon: x_{n}<t<y_{n}\right\}, \quad \Gamma_{-}=\left\{t-i \epsilon: x_{n}<t<y_{n}\right\} \\
& \Gamma_{x_{n}}=\left\{x_{n}+i t:|t| \leq \epsilon\right\}, \quad \Gamma_{y_{n}}=\left\{y_{n}+i t:|t| \leq \epsilon\right\}
\end{aligned}
$$

which is a box with vertices at $\left(x_{n}, \epsilon\right),\left(x_{n},-\epsilon\right),\left(y_{n}, \epsilon\right)$, and $\left(y_{n}, \epsilon\right)$. We can count the zeros in a neighborhood of the negative axis by

$$
\left|\left\{\Re z \in(y, x):|\Im z| \leq \epsilon, Q_{n}(z)=0\right\}\right|=\frac{1}{2 \pi i} \int_{\Gamma_{\epsilon}} \frac{Q_{n}^{\prime}(z)}{Q_{n}(z)} d z .
$$

Using this strategy we decompose our contour into four separate integrals

$$
\frac{1}{2 \pi i} \int_{\Gamma_{\epsilon}} \frac{Q_{n}^{\prime}(z)}{Q_{n}(z)} d z=\frac{1}{2 \pi i}\left(\int_{\Gamma_{+}}-\int_{\Gamma_{-}}+\int_{\Gamma_{x_{n}}}-\int_{\Gamma_{y_{n}}}\right) \frac{Q_{n}^{\prime}(z)}{Q_{n}(z)} d z .
$$

A direct application of Theorem 14 gives the contribution along the contours $\Gamma_{ \pm}$: "major contours:"

\section{Corollary 35.}

$$
\begin{aligned}
& \lim _{n \rightarrow \infty} \frac{1}{2 \pi i n^{\frac{2}{3}}} \int_{\Gamma_{+}} \frac{Q_{n}^{\prime}(z)}{Q_{n}(z)} d z=\frac{3}{2 \pi} \frac{\left(L_{1}(y+i \epsilon)-L_{1}(x+i \epsilon)\right)}{2 i} \\
& \lim _{n \rightarrow \infty} \frac{1}{2 \pi i n^{\frac{2}{3}}} \int_{\Gamma_{-}} \frac{Q_{n}^{\prime}(z)}{Q_{n}(z)} d z=\frac{3}{2 \pi} \frac{\left[L_{1}(y+i \epsilon)-L_{1}(x+i \epsilon)\right]^{-}}{2 i}
\end{aligned}
$$

Then we estimate the two remaining contours $\Gamma_{x_{n}}$ and $\Gamma_{y_{n}}$ ("minor contours") by the Bäcklund method of estimating the number of zeros of the zeta function (see [5] for an exposition).

Lemma 36. (a) If $W_{n}^{\prime}$ denotes the number of zeros of $\Re Q_{n}\left(x_{n}+i t\right)$ on the interval $t \in[0, \epsilon]$ then

$$
\left|\frac{1}{2 \pi i n^{\frac{2}{3}}} \int_{\Gamma_{x_{n}}} \frac{Q_{n}^{\prime}(x)}{Q_{n}(x)} d x\right| \leq \frac{W_{n}^{\prime}}{n^{\frac{2}{3}}} .
$$


(b) If $V_{n}^{\prime}$ denotes the number of zeros of $\Re Q_{n}\left(y_{n}+i t\right)$ on the interval $t \in[0, \epsilon]$, then

$$
\left|\frac{1}{2 \pi i n^{\frac{2}{3}}} \int_{\Gamma_{y_{n}}} \frac{Q_{n}^{\prime}(x)}{Q_{n}(x)} d x\right| \leq \frac{V_{n}^{\prime}}{n^{\frac{2}{3}}}
$$

Lemma 37.

$$
\begin{aligned}
& \limsup _{n \rightarrow \infty} \frac{1}{n^{\frac{2}{3}}} W_{n}^{\prime} \leq 3 \sup _{|z|=2 \epsilon}\left(\Re L_{1}(x+i z)-\Re L_{1}(x)\right) \\
& \limsup _{n \rightarrow \infty} \frac{1}{n^{\frac{2}{3}}} V_{n}^{\prime} \leq 3 \sup _{|z|=2 \epsilon}\left(\Re L_{1}(y+i z)-\Re L_{1}(y)\right) .
\end{aligned}
$$

Proof. Because the same estimates work for $V_{n}^{\prime}$ almost verbatim, we only will estimate $W_{n}^{\prime}$. To do so, define

$$
R Q_{n}(z)=\frac{1}{2}\left[Q_{n}\left(x_{n}-i z\right)+Q_{n}\left(x_{n}+i z\right)\right]
$$

The function $R Q_{n}(z)$ is an analytic function such that when $z$ is real, $R Q_{n}(z)=\Re Q_{n}\left(x_{n}+\right.$ $i z)$. We let $W_{n}$ denote the number of zeros $R Q_{n}(z)$ inside $B\left(x_{n}, \epsilon\right)$. Obviously $W_{n}^{\prime}<W_{n}$.

In [12], they use a standard consequence of Jensen's formula: suppose that $h(x)$ is an analytic function that has $m$ zeros inside $B(a, r)$. If $R>r$ and $h(a) \neq 0$, then $m \leq \max _{|\zeta|=R}(\ln |h(a+\zeta)-\ln h(a)|) /(\ln R-\ln r)$. To estimate $W_{n}$, we take $R=2 \epsilon$ and $r=\epsilon$ to obtain

$$
W_{n} \leq \frac{\sup _{|z|=2 \epsilon}\left(\ln \left|R Q_{n}(z) / R Q_{n}(0)\right|\right)}{\ln 2} \leq 2 \sup _{|z|=2 \epsilon}\left(\ln \left|\frac{Q_{n}\left(x_{n}+i z\right)}{Q_{n}\left(x_{n}\right)}\right|\right) .
$$

We then apply Theorem 15 . Because $x_{n} \in S_{n}$, given by Lemma 34, our estimate simplifies to

$$
Q_{n}\left(x_{n}\right)=2 \sqrt[12]{1-x_{n}}\left[\sqrt{\frac{\left|L_{1}\left(x_{n}\right)\right|}{n^{\frac{4}{3}} 6 \pi}} \exp \left(n^{\frac{2}{3}}\left(\frac{3}{2} \Re L_{1}\left(x_{n}\right)\right)\right)\right]^{-}\left(1+O_{X}\left(\frac{1}{n^{\frac{1}{3}}}\right)\right),
$$

which allows us to write

$$
\ln Q_{n}\left(x_{n}\right)=\frac{3}{2} n^{\frac{2}{3}} \Re L_{1}\left(x_{n}\right)+O_{x}(\ln n) .
$$

To estimate $Q_{n}(x)$ uniformly on a neighborhood of $x_{n}$ we invoke another theorem from [7]: If $X \subset \mathbb{D}$ is compact then we have the uniform estimate:

$$
Q_{n}(x)=O_{X}\left(e^{\frac{3}{2} n^{\frac{2}{3}} \max \left[\Re L_{1}(x), \Re L_{2}(x)\right]}\right) .
$$

Because $x_{n} \in\left(x^{*}, 0\right) \subset R(1)$ for sufficiently small $\epsilon>0$, we can make the bound

$$
2 \sup _{|z|=2 \epsilon}\left(\ln \left|Q_{n}\left(x_{n}+i z\right) / Q_{n}\left(x_{n}\right)\right|\right) \leq 3 n^{\frac{2}{3}} \sup _{|z|=2 \epsilon}\left(\Re L_{1}\left(x_{n}+i z\right)-\Re L_{1}\left(x_{n}\right)\right)+O_{x}(\ln n) .
$$


Conclude the proof of the lemma by taking the limit

$$
\lim _{n \rightarrow \infty} \frac{1}{n^{\frac{2}{3}}} W_{n} \leq 3 \sup _{|z|=2 \epsilon}\left(\Re L_{1}(x+i z)-\Re L_{1}(x)\right) .
$$

We now finish the proof of our density result. Combining Lemmas 36 and 37, we obtain the bounds for the contours $\Gamma_{x_{n}}$ and $\Gamma_{y_{n}}$ :

$$
\begin{aligned}
& \lim _{n \rightarrow \infty} \frac{1}{2 \pi i n^{\frac{2}{3}}} \int_{\Gamma_{x_{n}}} \frac{Q_{n}^{\prime}(x)}{Q_{n}(x)} d x \leq 3 \sup _{|z|=2 \epsilon}\left(\Re L_{1}(x+i z)-\Re L_{1}(x)\right)=O_{x}(\epsilon), \\
& \lim _{n \rightarrow \infty} \frac{1}{2 \pi i n^{\frac{2}{3}}} \int_{\Gamma_{y_{n}}} \frac{Q_{n}^{\prime}(x)}{Q_{n}(x)} d x \leq 3 \sup _{|z|=2 \epsilon}\left(\Re L_{1}(y+i z)-\Re L_{1}(y)\right)=O_{y}(\epsilon) .
\end{aligned}
$$

Substituting in all of our estimates, we obtain

$$
\lim _{n \rightarrow \infty} \frac{1}{n^{\frac{2}{3}}} \frac{1}{2 \pi i} \int_{\Gamma_{\epsilon}} \frac{Q_{n}^{\prime}(z)}{Q_{n}(z)} d z=\frac{3}{2 \pi} \Im\left[\left(L_{1}(y+i \epsilon)-L_{1}(x+i \epsilon)\right)\right]+O_{x, y}(\epsilon) .
$$

We complete the proof by taking the infimum over $\epsilon>0$.

Remark 38. For any compact subset $X$ on $\left(x^{*}, 0\right)$, we see that the zeros of $Q_{n}(x)$ there satisfy the equation

$$
\begin{aligned}
0= & \sqrt[12]{1-x}\left[\sqrt{\frac{L_{1}(x)}{n^{\frac{4}{3}} 6 \pi}} \exp \left(n^{\frac{2}{3}}\left(\frac{3}{2} L_{1}(x)\right)\right)\right]^{-}\left(1+O_{X}\left(\frac{1}{n^{\frac{1}{3}}}\right)\right) \\
& +\sqrt[12]{1-x}\left[\sqrt{\frac{L_{1}(x)}{n^{\frac{4}{3}} 6 \pi}} \exp \left(n^{\frac{2}{3}}\left(\frac{3}{2} L_{1}(x)\right)\right)\right]\left(1+O_{X}\left(\frac{1}{n^{\frac{1}{3}}}\right)\right),
\end{aligned}
$$

which simplifies to

$$
0=\cos \left(\frac{3 \sqrt{3}}{4} n^{\frac{2}{3}} \sqrt[3]{2}\left(-L i_{3}(x)\right)^{1 / 3}+\frac{\pi}{6}\right)+O_{X}\left(n^{-1 / 3}\right)
$$

since $\operatorname{Li}_{3}(x)$ is negative on $X$ the argument of $L_{1}(x)$ is constant. This last equation provides a good quantitative estimate for the roots of the polynomial on $X$ by deleting the big-oh contribution:

$$
\cos \left(\frac{3 \sqrt{3}}{4} n^{\frac{2}{3}} \sqrt[3]{2}\left(-L i_{3}(x)\right)^{1 / 3}+\frac{\pi}{6}\right)=0 .
$$

In Figure 5, we plot $-\log _{10}$ of the relative error e two ways so in both plots the vertical axis measures the number of significant digits of accuracy. In the first plot, we simply plot $-\log _{10}(\mathbf{e})$ listing roots in a uniform fashion while in the second plot we plot $-\log _{10}(\mathbf{e})$ above the actual root on the negative axis. To find the numerical values for 

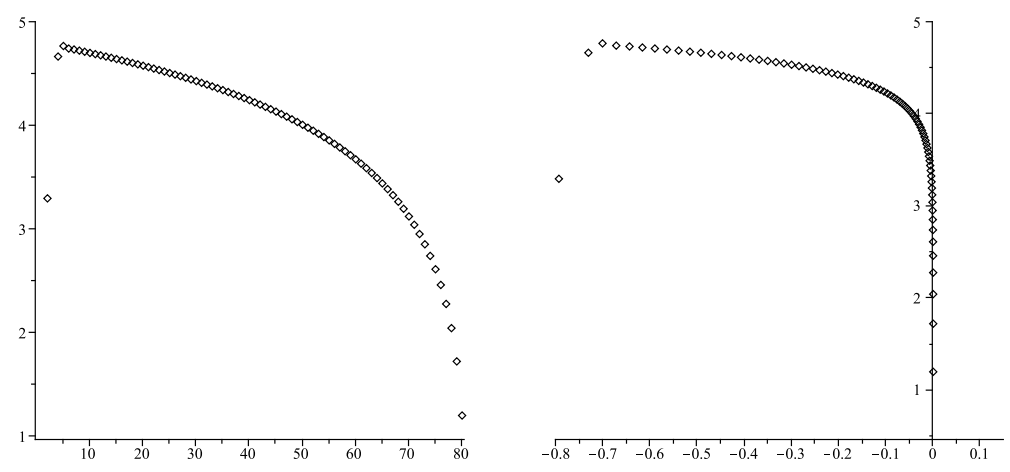

Figure 5: Two views of the relative error of using the approximation of the zero of the asymptotic estimate instead of the actual root of $Q_{2200}(x)$. The vertical scale represents the number of significant digits of accuracy.

the 79 negative zeros $Q_{2200}(x)$, we used the software MPSolve [2] while for solving the nonlinear equation from the asymptotic estimate we used the command fsolve in Maple.

We observe that the results of this section are much stronger than simply knowing that the zero attractor $A$ contains the interval $\left[x^{*}, 0\right]$ since the zeros of $Q_{n}(x)$ may be non-real but still accumulate in the limit along a real interval while equation 4 guarantees that $Q_{n}(x)$ has zeros in the compact subset $X$ of $\left(x^{*}, 0\right)$ for $n$ sufficiently large.

\section{Appendix: Proof of Lemma 30}

In Figure 6, we see strong numerical evidence for the inequality of the lemma.
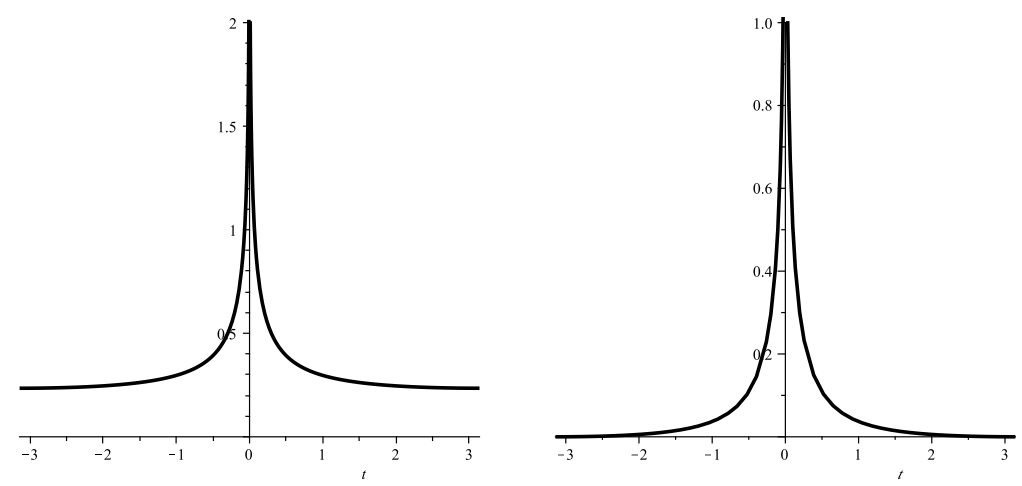

Figure 6: On left plot is the plot of $\Re\left(\frac{L i_{1}(x)}{L i_{2}(x)}-\frac{2}{3} \frac{L i_{2}(x)}{L i_{3}(x)}\right)$ where $x=e^{i \theta}$ and $\theta \in(-\pi, \pi)$. On the right, the constant $2 / 3$ is changed to $3 \zeta(3) \ln (2) / \zeta(2)^{2}$. 
Proof. By the minimum principle for harmonic functions, it is sufficient to establish the inequality on the unit circle. Let

$$
f(\theta)=\Re\left(\frac{L i_{1}\left(e^{i \theta}\right)}{L i_{2}\left(e^{i \theta}\right)}-\frac{2}{3} \frac{L i_{2}\left(e^{i \theta}\right)}{L i_{3}\left(e^{i \theta}\right)}\right) .
$$

Note that $f$ is an even function and $f(\theta) \rightarrow \infty$ as $\theta \rightarrow 0^{+}$. So it is enough to show that $f(\theta)>0$ on $(0, \pi]$. Since $\left|L i_{s}\left(e^{i \theta}\right)\right|$ is a decreasing function of $\theta[6$, Section 2], we have the bounds

$$
\left(1-2^{1-s}\right) \zeta(s)=L i_{s}(-1) \leq\left|L i_{s}\left(e^{i \theta}\right)\right| \leq \zeta(s) .
$$

In particular, we find that $\frac{1}{2} \zeta(2) \leq\left|L i_{2}\left(e^{i \theta}\right)\right| \leq \zeta(2)$ and $\frac{3}{4} \zeta(3) \leq\left|L i_{3}\left(e^{i \theta}\right)\right| \leq \zeta(3)$ on $[0, \pi]$.

We first show that there exists $\theta_{0} \in(0, \pi)$ such that $f(\theta)>0$ on $\left(0, \theta_{0}\right)$. Now we have the lower bound

$$
\Re\left(\frac{L i_{1}\left(e^{i \theta}\right)}{L i_{2}\left(e^{i \theta}\right)}-\frac{2}{3} \frac{L i_{2}\left(e^{i \theta}\right)}{L i_{3}\left(e^{i \theta}\right)}\right) \geq \Re\left(\frac{L i_{1}\left(e^{i \theta}\right)}{L i_{2}\left(e^{i \theta}\right)}\right)-\frac{2}{3} \frac{\zeta(2)}{\frac{3}{4} \zeta(3)} .
$$

On $(0, \pi]$, we can simplify further

$$
\begin{aligned}
\Re\left(\frac{L i_{1}\left(e^{i \theta}\right)}{L i_{2}\left(e^{i \theta}\right)}\right) & =\Re\left(\frac{-\ln \left(1-e^{i \theta}\right)}{L i_{2}\left(e^{i \theta}\right)}\right) \\
& =-\ln \left|1-e^{i \theta}\right| \Re\left(\frac{1}{L i_{2}\left(e^{i \theta}\right)}\right)+\arg \left(1-e^{i \theta}\right) \Im\left(\frac{1}{L i_{2}\left(e^{i \theta}\right)}\right) \\
& \geq-\ln \left|1-e^{i \theta}\right| \Re\left(\frac{1}{L i_{2}\left(e^{i \theta}\right)}\right)-\frac{2 \pi}{\zeta(2)} \\
& =-\ln \left|1-e^{i \theta}\right| \frac{1}{\left|L i_{2}\left(e^{i \theta}\right)\right|} \cos \left(\arg \left(L i_{2}\left(e^{i \theta}\right)\right)\right)-\frac{2 \pi}{\zeta(2)} .
\end{aligned}
$$

By [13], we know that $L i_{2}(x)$ is convex and therefore a starlike function. Recall that for any starlike function $g(x)$ if $x=e^{i \theta}$ we have

$$
\Re\left(x \frac{g^{\prime}(x)}{g(x)}\right)=\frac{d}{d \theta} \arg g\left(e^{i \theta}\right)>0 .
$$

In particular, $\arg L i_{2}\left(e^{i \theta}\right)$ is an increasing function of $\theta$.

For any $\theta_{1}$ such that $0<\arg L i_{2}\left(e^{i \theta_{1}}\right)<\pi / 2$, we know that $\cos \arg L i_{2}\left(e^{i \theta}\right)$ is decreasing on $\left(0, \theta_{1}\right)$ so is bounded below by $\cos \arg L i_{2}\left(e^{i \theta_{1}}\right)$. So we obtain an refinement of the above lower bound on $\left(0, \theta_{1}\right)$

$$
\Re\left(\frac{L i_{1}\left(e^{i \theta}\right)}{L i_{2}\left(e^{i \theta}\right)}\right) \geq-\ln \left|1-e^{i \theta}\right| \frac{1}{\left|L i_{2}\left(e^{i \theta}\right)\right|} \cos \left(\arg \left(L i_{2}\left(e^{i \theta_{1}}\right)\right)\right)-\frac{2 \pi}{\zeta(2)}
$$

which leads to the lower bound for $f(\theta)$

$$
f(\theta) \geq-\ln \left|1-e^{i \theta}\right| \frac{1}{\zeta(2)} \cos \left(\arg \left(L i_{2}\left(e^{i \theta_{1}}\right)\right)\right)-\frac{2 \pi}{\zeta(2)}-\frac{8}{9} \frac{\zeta(2)}{\zeta(3)}
$$


We now restrict to $\theta$ such that

$$
-\ln \left|1-e^{i \theta}\right| \cos \left(\arg \left(L i_{2}\left(e^{i \theta_{1}}\right)\right)\right)>2 \pi+\frac{8}{9} \frac{\zeta(2)^{2}}{\zeta(3)} .
$$

This is equivalent to

$$
\cos \theta>1-\frac{1}{2} \exp \left(-2 \sec \left(\arg \left(L i_{2}\left(e^{i \theta_{1}}\right)\right)\right)\left[2 \pi+\frac{8}{9} \frac{\zeta(2)^{2}}{\zeta(3)}\right]\right) .
$$

Consequently, for $\theta<\pi / 2$, we have

$$
\theta<\arccos \left(1-\frac{1}{2} \exp \left(-2 \sec \left(\arg \left(L i_{2}\left(e^{i \theta_{1}}\right)\right)\right)\left[2 \pi+\frac{8}{9} \frac{\zeta(2)^{2}}{\zeta(3)}\right]\right)\right)=\theta_{2} .
$$

Hence $f(\theta)>0$ on $\left(0, \theta_{0}\right)$ where

$$
\theta_{0}=\min \left[\theta_{1}, \theta_{2}, \theta_{3}\right]
$$

where $\theta_{3} \simeq 1.327793289 \ldots$ is chosen so $\arg L i_{2}\left(e^{i \theta_{3}}\right)=\pi / 2$. We find that $\theta_{0}$ may be taken as $2 \times 10^{-5}$.

We now turn to establishing the inequality on $\left[\theta_{0}, \pi\right]$. Now $f(\theta)$ is differentiable and

$$
f^{\prime}(\theta)=-\Im\left(\frac{e^{i \theta}}{\left(1-e^{i \theta}\right) L i_{2}\left(e^{i \theta}\right)}-\left(\frac{\ln \left(1-e^{i \theta}\right)}{L i_{2}\left(e^{i \theta}\right)}\right)^{2}+\frac{\ln \left(1-e^{i \theta}\right)}{L i_{2}\left(e^{i \theta}\right)}+\frac{2}{3}\left(\frac{L i_{2}\left(e^{i \theta}\right)}{L i_{3}\left(e^{i \theta}\right)}\right)\right)
$$

We need a uniform bound for $\left|f^{\prime}\left(e^{i \theta}\right)\right|$ on $\left[\theta_{0}, \pi\right]$. We begin with the bound

$$
\left|\frac{e^{i \theta}}{\left(1-e^{i \theta}\right) L i_{2}\left(e^{i \theta}\right)}\right| \leq \frac{\pi}{\theta_{0} \zeta(2)}
$$

Since $\left|\ln \left(1-e^{i \theta}\right)\right|$ is decreasing on $(0, \pi)$, we have

$$
\left|\frac{\ln \left(1-e^{i \theta}\right)}{L i_{2}\left(e^{i \theta}\right)}\right| \leq 2 \frac{\left|\ln \left(1-e^{i \theta_{0}}\right)\right|}{\zeta(2)}
$$

Hence, we have the upper bound

$$
\begin{aligned}
\left|f^{\prime}(\theta)\right| & \leq \frac{\pi}{\theta_{0} \zeta(2)}+4 \frac{\left|\ln \left(1-e^{i \theta_{0}}\right)\right|^{2}}{\zeta(2)^{2}}+2 \frac{\left|\ln \left(1-e^{i \theta_{0}}\right)\right|}{\zeta(2)}+\frac{8}{9} \frac{\zeta(2)^{2}}{\zeta(3)^{2}} \\
& \simeq 95683.44241 \ldots \\
& \leq 95700 .
\end{aligned}
$$

To sum up, we have shown that $f(\theta)$ is a real-valued differentiable function on $\left[\theta_{0}, \pi\right]$ such that the magnitude of its derivative there is bounded by 95700. Suppose $f\left(\theta_{i}\right)>0$ for some $\theta_{i} \in\left[\theta_{0}, \pi\right]$. Then $f(\theta)>0$ for $\left|\theta-\theta_{i}\right| \leq f\left(\theta_{i}\right) / 194000$. This provides a computational method to finish the proof.

Let $\theta_{0}=2 \times 10^{-5}$. Note that $f(\theta)>0$ on $\left[\theta_{0}, \theta_{0}+f\left(\theta_{0}\right) / 19400\right]$. Next, given $\theta_{0}<\theta_{1}<$ $\cdots<\theta_{i-1}$ for $i \geq 1$ such that $f(\theta)>0$ on $\left[\theta_{0}, \theta_{i-1}\right]$, let $\theta_{i}=\theta_{i-1}+f\left(\theta_{i-1}\right) / 19400$. Then we have that $f$ is positive on $\left[\theta_{0}, \theta_{i}\right]$. The lemma holds provided there exists an integer $i$ such that $\theta_{i}>\pi$. Numerically, we verified that $f\left(\theta_{i}\right) \geq 0.2345735690$, for $0 \leq i \leq 2135923$, and $\theta_{2135924}=3.142000829>\pi$. So the proof is complete. 
Remark 39. We thank the referee for suggesting the above proof. The bound in Lemma 30 is not tight; for example, W. Zudilin [19] suggested the improvement of changing the coefficient $2 / 3$ to $3 \zeta(3) \ln (2) / \zeta(2)^{2} \simeq 0.9237931819$ that gives equality at $x=-1$. See Figure 6 .

\section{References}

[1] Patrick Billingsley, "Convergence of Probability Measures," Wiley, 1999.

[2] Dario Bini and Giuseppe Fiorentino, Design, analysis, and implementation of a multiprecision polynomial rootfinder, Numerical Algorithms, 23 (2000), 127-173.

[3] M. Biskup, C. Borgs, J. T. Chayes, and R. Kotecky, Partition function zeros at first-order phase transitions: A general analysis, Commun. Math. Phys, 116 (2004) $79-131$.

[4] Robert P. Boyer and William M. Y. Goh, Partition polynomials: asymptotics and zeros, In Tapas in experimental mathematics, Contemporary Mathematics, Volume 457, pages 99-111. Amer. Math. Soc., Providence, RI, 2008.

[5] Robert P. Boyer and William M. Y. Goh, Appell Polynomials and Their Zero Attractors, Contemporary Mathematics, Volume 517 (2010), 69-96.

[6] Robert P. Boyer and Daniel T. Parry. Phase calculations for plane partition polynomials, accepted for publication, Rocky Mountain Journal of Mathematics.

[7] Robert P. Boyer Daniel T. Parry, Plane partition polynomials and weighted plane partitions. submitted for publication, 2011.

[8] E. Rodney Canfield, Sylvie Corteel, and Carla D. Savage, Durfee polynomials, Electron. J. Combin., 5 (1998) \#R32 (21 pages).

[9] John Conway, "Functions of One Complex Variables" Graduate Texts in Mathematics Vol. 11, Springer-Verlag, New York, NY, 1978.

[10] Peter L. Duren, "Univalent Functions," Grundlehren der mathematischen Wissenschaften Vol. 259, Springer-Verlag, New York, NY, 1983.

[11] Paul Erdös and P. Turán, On the distribution of roots of polynomials, Ann. Math. (2) 51 (1950) 105-119.

[12] William M. Y. Goh, Matthew He, and Paolo Ricci, On the universal zero attractor of the Tribonacci-related polynomials, Calcolo 46 (2009) 95-129.

[13] John L. Lewis, Convexity of a Certain Series, J. London Math. Society (2), 27 (1983) 435-446.

[14] Athanase Papadopoulos, "Metric Spaces, Convexity and Nonpositive Curvature," European Mathematical Society, 2005.

[15] Alan Sokal, Chromatic roots are dense in the whole complex plane, Combinatorics, Probability \& Computing 13 (2004), 221-261.

[16] Richard P. Stanley, http://www-math.mit.edu/ rstan/zeros 
[17] E. M. Wright, Asymptotic Partition Formulae: (I) Plane Partitions, Quart. J. Math. Oxford Ser. (2) 2 (1931) 177-189.

[18] E. M. Wright, Asymptotic Partition Formulae: (II) Weighted Partitions, Proceedings of the London Mathematical Society (2) 36 (1933) 117-141.

[19] Wadim Zudilin, http://mathoverflow.net/questions/54609 (version: 2011-02-07) 